\title{
5-Hydroxymethylcytosine expression in metastatic melanoma versus nodal nevus in sentinel lymph node biopsies
}

\author{
Jonathan J Lee ${ }^{1}$, Scott R Granter ${ }^{1}$, Alvaro C Laga ${ }^{1}$, Arturo P Saavedra ${ }^{1,2}$, Qian Zhan ${ }^{1}$, \\ Weimin Guo ${ }^{1}$, Shuyun $\mathrm{Xu}^{1}$, George F Murphy ${ }^{1}$ and Christine G Lian ${ }^{1}$ \\ ${ }^{1}$ Program in Dermatopathology, Department of Pathology, Brigham and Women's Hospital, Harvard Medical \\ School, Boston, MA, USA and ${ }^{2}$ Department of Dermatology, Massachusetts General Hospital, Harvard \\ Medical School, Boston, MA, USA
}

\begin{abstract}
Sentinel lymph node biopsies are conducted to stage patients with newly diagnosed melanomas that have histopathological attributes conferring defined levels of metastatic potential. Because benign nevic cells may also form 'deposits' in lymph nodes (nodal nevus), the pathological evaluation for metastatic melanoma within sentinel lymph nodes can be challenging. Twenty-eight sentinel lymph node biopsy cases containing either metastatic melanoma $(N=18)$ or nodal nevi $(N=10)$ were retrieved from the archives of the Brigham and Women's Hospital, Department of Pathology (2011-2014). In addition, two sentinel lymph node cases that were favored to represent metastatic disease but whose histopathological features were viewed as equivocal, with melanoma favored, were also included. Dual labeling for the melanocyte lineage marker, MART-1, and the epigenetic marker, 5-hydroxymethylcytosine, a functionally significant indicator that has been shown to distinguish benign nevi from melanoma, was performed on all cases using immunohistochemistry and/or direct immunofluorescence. All (18 of 18) metastatic melanoma cases showed complete loss of 5-hydroxymethylcytosine nuclear staining in MART-1-positive cells, and all (10 of 10) nodal nevus cases demonstrated 5-hydroxymethylcytosine nuclear staining in MART-1-positive cells. In addition, 5-hydroxymethylcytosine staining confirmed the favored diagnoses of metastatic melanoma in the two 'equivocal' cases. Thus, 5-hydroxymethylcytosine may be a useful adjunctive marker to distinguish between benign nodal nevi and metastatic melanoma during the evaluation of sentinel lymph node biopsies for metastatic melanoma.

Modern Pathology (2015) 28, 218-229; doi:10.1038/modpathol.2014.99; published online 1 August 2014
\end{abstract}

Melanoma patients with defined histopathological attributes in their primary lesions undergo sentinel lymph node biopsy in accordance with the American Joint Commission on Cancer (AJCC) guidelines. ${ }^{1}$ The implications of an accurate sentinel lymph node evaluation are not trivial, as completion lymphadenectomy, a procedure with significant potential for morbidity, generally follows a positive sentinel lymph node diagnosis. During the evaluation of such sentinel lymph node biopsies, however, benign nodal nevi (also termed 'nodal melanocytic nevi', 'intranodal nevus', 'nevic rest', 'nevus cell aggregates', and 'lymph node nevus') are

Correspondence: Dr GF Murphy, MD or Dr CG Lian, MD, Program in Dermatopathology, Department of Pathology, Brigham and Women's Hospital, Harvard Medical School, 221 Longwood Avenue, EBRC Suite 401, Boston, MA 02115, USA.

E-mail: gmurphy@rics.bwh.harvard.edu or cglian@partners.org Received 15 May 2014; revised 22 May 2014; accepted 23 May 2014; published online 1 August 2014 frequently encountered in routine practice, representing one of the major diagnostic pitfalls. Some studies suggest that up to $22 \%$ of sentinel lymphadenectomy specimens from patients with melanoma may contain nodal nevi. ${ }^{2}$

There are two prevailing theories to explain the origin of nevic cell deposits within lymph nodes. The first theory, termed 'emoblic transfer' (also referred to as 'mechanical transport' or 'benign metastasis'), suggests that nevic cells from benign cutaneous lesions gain entry into the lymphatics and are 'embolically' transferred to draining lymph nodes. ${ }^{2}$ There are several observations in support of this theory. It is known that the presence of nodal nevi associates tightly with the presence of cutaneous nevi in corresponding anatomic catchment areas. ${ }^{3}$ This association is even stronger with nevi of the congenital type, including those that grow in intimate spatial relationship with adnexal, neural, and lymphovascular structures. ${ }^{3}$ In addition, patients with nevi in draining nodes have been reported to be 
significantly more likely to have had a primary cutaneous melanoma arising in association with a preexisting nevus. ${ }^{2}$ This observation has led some authors to hypothesize that benign nevic cells may be 'displaced' by their adjacent melanoma and, thereby, gain entry into the lymphatics. ${ }^{2}$

The second theory proposes that nodal nevi arise from the arrested migration of neural crest progenitor cells during embryological development. ${ }^{3}$ The fact that nodal nevi have been documented in catchment areas devoid of cutaneous nevi may support this hypothesis, although it is known that the natural history of common nevi is to undergo senescence and ultimately disappear, and the majority of cutaneous nevi are acquired (ie, not present at birth). ${ }^{2}$ Recent studies demonstrate that the overall 5-year survival for patients with nodal nevi does not differ significantly from that of patients with negative sentinel lymph nodes. ${ }^{4}$ In contrast, positivity for metastatic melanoma is both an ominous and powerful predictor of disease progression and overall survival. ${ }^{1}$ Thus, distinguishing between these two entities is a matter of clinical, pathological, and prognostic import.

Despite conventional dogma suggesting that nodal nevi reside within the lymph node capsule or trabeculae, whereas metastatic melanomas are subcapsular, sinusoidal, or intraparenchymal, ${ }^{2}$ such features may be inadequate for making a definitive distinction in routine practice. Cytomorphology may also be useful, although factors such as the cellular enlargement of nevus cells due to activation or, conversely, the 'small cell' differentiation of melanoma cells may limit the use of this parameter. ${ }^{5}$ This is particularly true, given that a subset of melanomas not infrequently exhibit a deceivingly bland cytomorphology. In addition, routine immunohistochemical evaluation with melanocytic markers (ie, S-100, MART-1, Melan-A, and SOX-10) simply cannot distinguish a benign nodal nevus from metastatic melanoma, nor can HMB-45, which is also expressed in substantial subset of nodal nevi. ${ }^{6}$

A number of markers have been reported in the literature to aid in making the histopathological distinction between nodal nevi and metastatic melanoma in sentinel lymph nodes. The immunohistochemical detection of $p 16$, a component of the tumor suppressor gene involved in negative cell cycle regulation and known to be lost in familial melanoma, has been suggested to be useful in distinguishing between nodal nevi and metastatic melanoma in sentinel lymph node biopsies. ${ }^{7}$ However, more recent studies demonstrate that $p 16$ does not consistently distinguish between certain melanomas and benign nevi in primary skin sections. ${ }^{8}$ For this reason, p16 may not be as reliable or practical of a marker in routine practice. In addition, the use of fluorescence in situ hybridization (FISH) targeting specific regions on chromosomes 6 and 11 to detect the presence of certain chromosomal aberrations has also been reported to distinguish metastatic melanoma from nodal nevi. ${ }^{9}$ The routine use of FISH to distinguish between these two entities, however, has practical and economic limitations. Finally, we have reported that the neural crest embryonic stem cell transcription factor, SOX2, to be a novel biomarker that discriminates melanoma from nevi, ${ }^{10}$ functionally contributes to melanoma invasion, ${ }^{11}$ and exhibits biological interplay with the intermediate filament protein, nestin. ${ }^{10}$ Chen et $a l^{5}$ have recently shown that the combined use of SOX2 and nestin has predictive value in differentiating nevi from melanoma in sentinel lymph nodes, underscoring the feasibility and importance of continued and aggressive exploration of functionally relevant biomarkers that will be useful adjuncts in sentinel lymph node evaluation.

We have previously shown that benign cutaneous nevi retain high levels of nuclear staining for the epigenetic hallmark, 5-hydroxymethylcytosine, whereas dysplastic nevi and primary malignant melanomas demonstrate partial to complete loss, respectively. ${ }^{12,13}$ Biologically, this 'loss of 5-hydroxymethylcytosine' reflects dysfunction of the Ten-Eleven Translocase (TET) family of 5-methylcytosine hydroxylases that converts 5-methylcytosine to 5-hydroxymethylcytosine, and, thereby, ultimately enables active DNA demethylation. ${ }^{14}$ This critical regulatory function has earned TET the epithet, 'guardian' of DNA methylation fidelity. ${ }^{15}$ Thus, the absence of 5-hydroxymethylcytosine may be a rather sensitive and specific marker for distinguishing benign from malignant melanocytic cells. Accordingly, herein we explore the clinical application of dual labeling with MART1 and 5-hydroxymethylcytosine to aid in the distinction between nodal nevi and metastatic melanoma in sentinel lymph node biopsies.

\section{Materials and methods}

\section{Histopathological Samples}

This study was approved by the Institutional Review Board of the Brigham and Women's Hospital. A total of 28 sentinel lymph node biopsy cases containing either histologically confirmed metastatic melanoma $(N=18)$ or nodal nevi $(N=10)$ were retrieved from the archives of the Brigham and Women's Hospital Department of Pathology (20112014). There were no sentinel lymph node biopsy cases in which one lymph node contained metastatic melanoma and another contained a nodal nevus. In addition, two 'equivocal' sentinel lymph node cases, whose diagnoses were debated, were also included in our study. One case (case 1) featured scattered intraparenchymal MART-1-positive cells that contained minimally atypical nuclei that were only slightly larger than those of lymphocytes and with minimal nuclear atypia. The second case (case 2) contained MART-1-positive 
cells predominantly within the capsule with regions consistent with involvement of an intracapsular angiolympatic space; these cells contained enlarged, somewhat atypical nuclei. Both cases resulted in extensive discussion among the dermatopathologists, with a consensus opinion favoring metastatic melanoma in both. H\&E and routine S-100 and MART-1-stained sections were independently reviewed, and the prior diagnoses were confirmed independently by two dermatopathologists (GFM and CGL). In addition, histopathological data of the sentinel lymph node biopsies, including features such as the anatomic site of the biopsy, the microanatomic location and size of the focus, and pattern of spread, among others, were also recorded.

\section{Immunohistochemistry and Immunofluorescence}

Immunohistochemistry was performed on all cases $(N=30)$. Sections were incubated overnight with a mixture of rabbit-anti-5-hydroxymethylcytosine (Active Motif, Carlsbad, CA, USA) and mouse-anti-MART-1 antibodies (Covance, Princeton, NJ, USA). The sections were washed and subsequently incubated with a mixture of secondary antibodies, including alkaline phosphatase-linked anti-rabbit IgG (Vector Laboratories, Burlingame, CA, USA) and peroxidase-linked antimouse IgG (Vector Laboratories). The sections were then treated with their corresponding substrate kits (Vector Laboratories). In addition, dual-labeling immunofluorescence was performed to complement immunohistochemistry as a means of two-channel identification of epitopes with nuclear staining of 5-hydroxymethylcytosine and membranous staining for MART-1. Instead of incubation with the secondary antibodies, these sections were incubated with a mixture of goat anti-rabbit IgG (Alexa Fluor, Grand Island, NY) and goat anti-mouse IgG (Alexa Fluor). Appropriate isotype-matched antibody controls and tissue controls were used for all experiments.

\section{Clinical/histopathological Data of Primary Cutaneous Melanoma}

In addition, corresponding clinical information and histopathological data of the patient's primary melanomas were also obtained for each sentinel lymph node case. Variables such as the patient's age, gender, location of primary, depth of primary melanoma (compiled based on initial biopsy and updated with re-excision data), mitotic rate, presence of ulceration, and subsequent development of metastasis among others were recorded.

\section{Quantitative Analysis}

The resulting quantitative and categorical data was analyzed using MedCalc version 13.2 (MedCalc Software, Ostend, Belgium). The primary melanoma clinical and histopathological data, as well as the sentinel lymph node histopathological data were sorted into two categories based on whether they were associated with a nodal nevus or with a metastatic melanoma. Quantitative variables (ie, age, Breslow's depth, no. of foci within the sentinel lymph node, and so on) were compared between the two groups using a two-sample $t$-test; categorical variables were compiled into proportions (ie, proportion of cases with ulceration in primary lesion, proportion of cases of that were intracapsular) and compared between the two groups using a twosample z-test. All $P$-values were two-sided, with a $P<0.05$ considered statistically significant.

\section{Results}

\section{Clinical and Histopathological Data of the Primary Cutaneous Melanomas}

Clinical and histopathological data of the primary cutaneous melanomas that led to sentinel lymph node biopsies containing the unequivocal nodal nevus or melanoma are summarized in Table 1 . Patients with metastatic melanoma and nodal nevi did not differ significantly in age, gender, location of primary tumors, presence of ulceration, mitotic activity, presence of tumor-infiltrating lymphocytes, cytomorphology, or eventually having distant or locoregional metastases (average follow-up time, 15 months for both groups combined; range, 1-49 months). In addition, only 1 (one) of 18 (6\%) primary melanomas with nodal metastases was associated with a preexisting/precursor nevus, in contrast to 3 of $10(30 \%)$ primary melanomas that were associated with nodal nevi (this difference did not reach statistical significance). However, cases of metastatic melanoma did have significantly deeper primary lesions (average Breslow's depth of $3.1 \mathrm{~mm}$ ) compared with cases of nodal nevi (average Breslow's depth of $1.2 \mathrm{~mm} ; P=0.005$ ). The primary melanoma data from the two 'equivocal' cases were subsequently included and comparatively analyzed, but did not influence the statistical significance of the findings above $(P=0.01)$.

\section{Histopathological Data of Sentinel Lymph Node Biopsies}

The histopathological data of the sentinel lymph node biopsies containing unequivocal nodal nevus or melanoma are summarized in Table 2. The most common location of melanoma metastasis in sentinel lymph nodes was within the lymph node parenchyma (intraparenchymal) in $83 \%$ (15 of 18) of cases, whereas a much smaller fraction of nodal nevi (30\%, or 3 of 10 cases) shared the same location $(P=0.017)$. In contrast, $60 \%$ (6 of 10) nodal nevi were located within the capsule (intracapsular) whereas only $11 \%$ (two of 18) metastatic melanomas 
Table 1 Summary of clinical and histopathological data of corresponding primary cutaneous melanomas

\begin{tabular}{|c|c|c|}
\hline & Nodal nevi & Metastatic melanoma \\
\hline Total no. of cases & 10 & 18 \\
\hline Average age (range) & $56(25-84)$ & $64(29-92)$ \\
\hline \multirow[t]{2}{*}{ Gender } & Male: $70 \%(7 / 10)$ & Male: $72 \%(13 / 18)$ \\
\hline & Female: $30 \%(3 / 10)$ & Female: $28 \%(5 / 18)$ \\
\hline \multirow[t]{5}{*}{ Location of primary } & $\mathrm{H} \& \mathrm{~N}: 10 \%(1 / 10)$ & H\&N: 22\% (4/18) \\
\hline & Upper extremity: 20\% (2/10) & Upper extremity: $22 \%(4 / 18)$ \\
\hline & Trunk: $40 \%(4 / 10)$ & Trunk: $44 \%(8 / 18)$ \\
\hline & Lower extremity: 30\% (3/10) & Lower extremity: $6 \%(1 / 18)$ \\
\hline & & Unknown: $6 \%(1 / 18)$ \\
\hline Average depth of primary (range) in mm & $1.2(0.5-2.1)$ & $3.1(0.98-8.5)^{*}$ \\
\hline Average mitotic rate per $\mathrm{mm}^{2}$ (range) & $4.7(1-15)$ & $8.3(0-21)$ \\
\hline$\%$ Cases with ulceration & $10 \%(1 / 10)$ & $33 \%(6 / 18)$ \\
\hline \% Cases with tumor-infiltrating lymphocytes & $40 \%(4 / 10)$ & $55 \%(10 / 18)$ \\
\hline$\%$ Cases with distant or locoregional metastases & 0 & $39 \%(7 / 18)$ \\
\hline Average follow-up time (range) in months & $17.1(2-34)$ & $9.7(1-49)$ \\
\hline \multirow[t]{3}{*}{ Cytomorphology } & Epithelioid: 50\% (5/10) & Epithelioid: $72 \%(13 / 18)$ \\
\hline & Spindle: $40 \%(4 / 10)$ & Spindle: $11 \%(2 / 18)$ \\
\hline & Spitzoid: $10 \%(1 / 10)$ & Not specified: $17 \%(3 / 18)$ \\
\hline \% Cases with preexisting/precursor nevus & $30 \%(3$ of 10$)$ & $6 \%(1$ of 18$)$ \\
\hline
\end{tabular}

${ }^{*} P$-value $<0.05$.

Table 2 Summary of histopathological data of sentinel lymph node biopsy cases

\begin{tabular}{|c|c|c|}
\hline & Nodal nevi & Metastatic melanoma \\
\hline \multirow[t]{6}{*}{ Anatomic location } & Submandibular: $0 \%$ (0 of 10 ) & Submandibular: 6\% (1 of 18) \\
\hline & Cervical (neck): $10 \%$ (1 of 10 ) & Cervical (neck): 28\% (5 of 18) \\
\hline & Supraclavicular: $0 \%$ (0 of 10) & Supraclavicular: 6\% (1 of 18) \\
\hline & Axillary: $60 \%$ (6 of 10 ) & Axillary: $50 \%$ (9 of 18 ) \\
\hline & Inguinal: $10 \%$ ( 1 of 10$)$ & Inguinal: $11 \%$ ( 2 of 18 ) \\
\hline & Femoral: $20 \%$ (2 of 10 ) & Femoral: 0\% (0 of 18) \\
\hline \multirow[t]{4}{*}{ Location of focus } & Intracapsular: $60 \%$ (6 of 10) & Intracapsular: $11 \%$ (2 of 18$)^{*}$ \\
\hline & Intratrabecular: $20 \%$ (2 of 10 ) & Intratrabecular: $0 \%$ (0 of 18) \\
\hline & Subcapsular: $0 \%$ (0 of 10) & Subcapsular: $39 \%$ (7 of 18) \\
\hline & Intraparenchymal: $30 \%$ (3 of 10 ) & Intraparenchymal: $83 \%$ (15 of 18$)^{*}$ \\
\hline Average no. of foci (range) & $1.2(1-3)$ & $1.7(1-6)$ \\
\hline Average size of focus, in mm (range) & $0.4(0.03-0.5)$ & $1.6(0.03-10)$ \\
\hline Cytomorphology & Epithelioid: $100 \%$ (10 of 10) & Epithelioid: 94\% (17 of 18) \\
\hline & & Spindled: 6\% (1 of 18) \\
\hline \multirow{2}{*}{ Pattern of spread } & Nodular (aggregated): 70\% (7 of 10) & Nodular (aggregated): $89 \%$ (16 of 18) \\
\hline & Scattered (single cell): $30 \%$ (3 of 10) & Scattered (single cell): $11 \%$ (2 of 18) \\
\hline
\end{tabular}

${ }^{*} P$-value $<0.05$.

were at least partially intracapsular $(P=0.021)$. Cases of metastatic melanoma and nodal nevi did not differ significantly in the average number of foci (nodal nevi: 1.2; metastatic melanoma: 1.7), average size of foci (nodal nevi: $0.4 \mathrm{~mm}$; metastatic melanoma: $1.6 \mathrm{~mm}$ ), cytomorphology, or pattern of spread.

\section{5-Hydroxymethylcytosine Expression in Sentinel Lymph Node Biopsies}

All (18 of 18) unequivocal metastatic melanomas showed complete loss of 5-hydroxymethylcytosine nuclear staining within MART-1-positive cells by both dual-labeling immunohistochemistry and immunofluorescence evaluation (Figure 1). In contrast, all (10 of 10) unequivocal nodal nevi showed strong diffuse nuclear staining for 5-hydroxymethylcytosine within MART-1-positive cells by both approaches (Figure 2). Both ('cases 1 and 2') 'equivocal' cases demonstrated loss of 5-hydroxymethylcytosine nuclear expression, supporting a diagnosis of metastasis in both instances (Figures 3 and 4). Occasional MART-1-negative cells expressing nuclear 5-hydroxymethylcytosine (usually lymphocytes) served as an internal positive control for loss of 5-hydroxymethylcytosine in nodal melanoma cells.

\section{Discussion}

Sentinel lymph node sampling is a standard staging procedure offered to patients with specific histopathological attributes in their primary cutaneous 


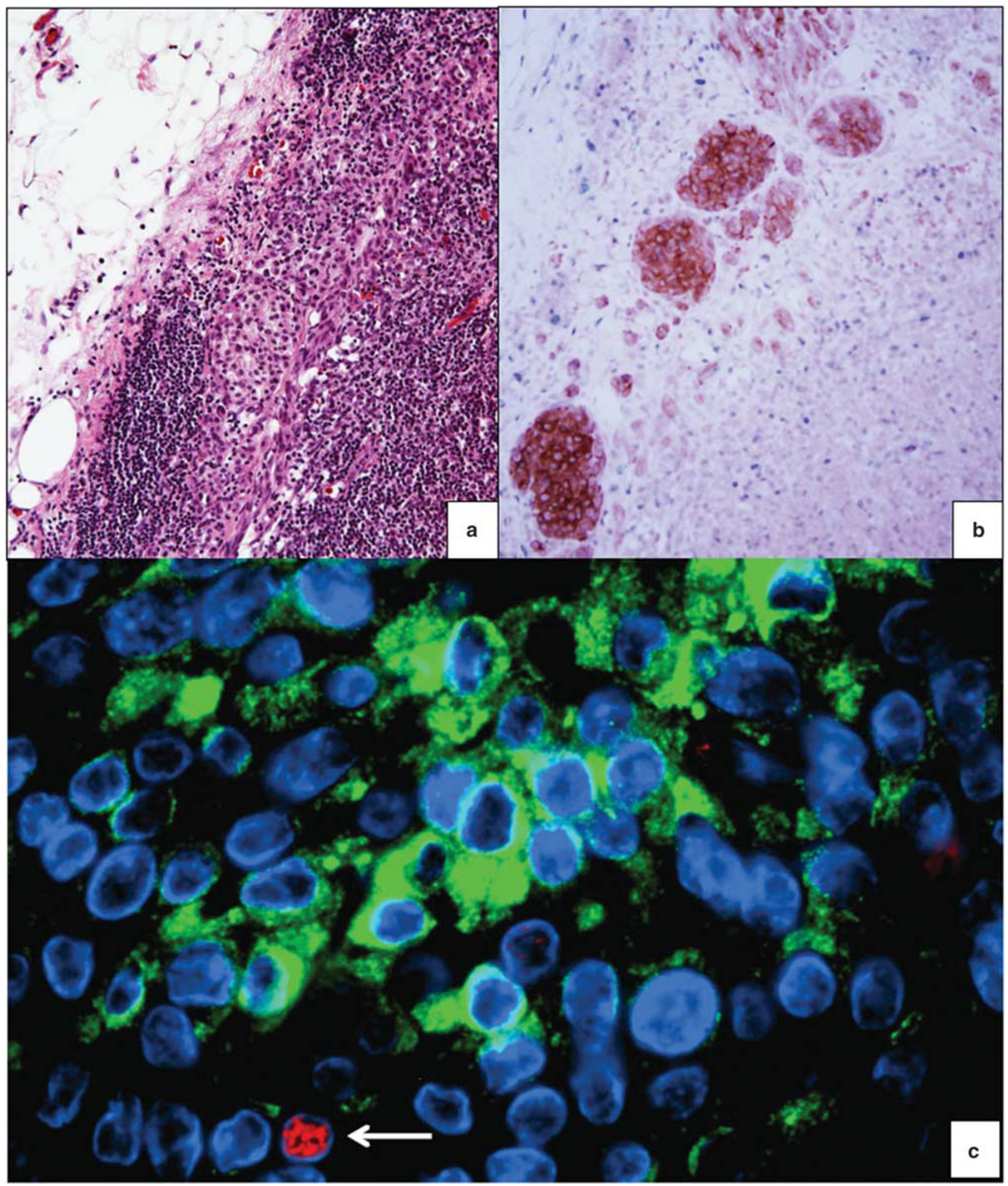

Figure 1 Metastatic melanoma. (a) Nests of epithelioid metastatic melanoma cells within the lymph node parenchyma (H\&E, $\times 20)$. (b) Dual-label IHC for MART-1 (brown, cytoplasmic) and 5-hydroxymethylcytosine (blue, nuclear) exhibits nests of MART-1-positive cells showing loss of nuclear 5-hydroxymethylcytosine expression $(\times 20)$. Note scattered mature lymphocytes showing 5hydroxymethylcytosine positivity. (c) Dual-label DIF for MART-1 and 5-hydroxymethylcytosine shows loss of 5-hydroxymethylcytosine (red, nuclear) within the MART-1 (green, cytoplasmic)-positive cells. Arrow indicates 5-hydroxymethylcytosine nuclear staining within lymphocyte, providing a positive internal control. Blue nuclear stain background provided by DAPI $(\times 100)$. 


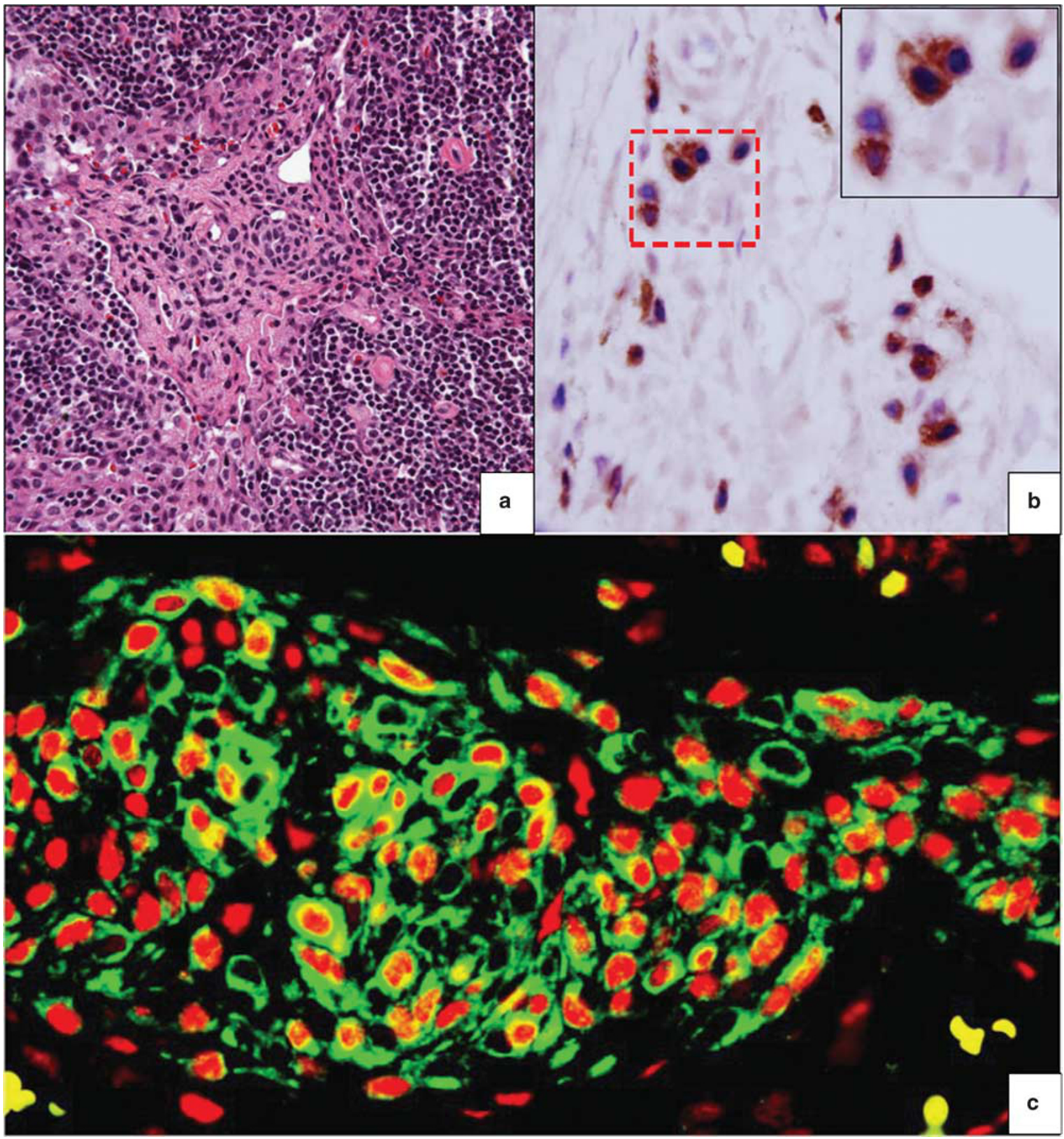

Figure 2 Nodal nevus. (a) Collection of bland-appearing melanocytes in nested pattern within lymph node trabeculum (H\&E, $\times 40)$. (b) Dual-label IHC for MART-1 (brown, cytoplasmic) and 5-hydroxymethylcytosine (blue, nuclear) demonstrates retention of 5-hydroxymethylcytosine expression within MART-1-positive cells $(\times 100$; inset: higher magnification for emphasis). (c) Dual-label DIF for MART-1 (green) and 5-hydroxymethylcytosine (red) demonstrates retention of 5-hydroxymethylcytosine nuclear stain within vast majority of MART-1-positive cells ( $\times 40)$.

melanoma. Most recently, the AJCC Melanoma Staging Committee and the National Comprehensive Cancer Network has expanded their criteria for considering sentinel lymph node biopsies to include those patients staged as T1b $(0.76-1.00 \mathrm{~mm}$ Breslow's depth with ulceration or mitoses $\geq 1 / \mathrm{mm}^{2}$ ) in addition to those staged as $\mathrm{T} 2$, $\mathrm{T} 3$, or $\mathrm{T} 44(>1.00 \mathrm{~mm}$ in thickness with or without ulceration, collectively). ${ }^{16}$ Despite the observed difference in average mitotic activity not reaching statistical significance within our cohort, patients having positive sentinel lymph nodes were, indeed, significantly more likely 


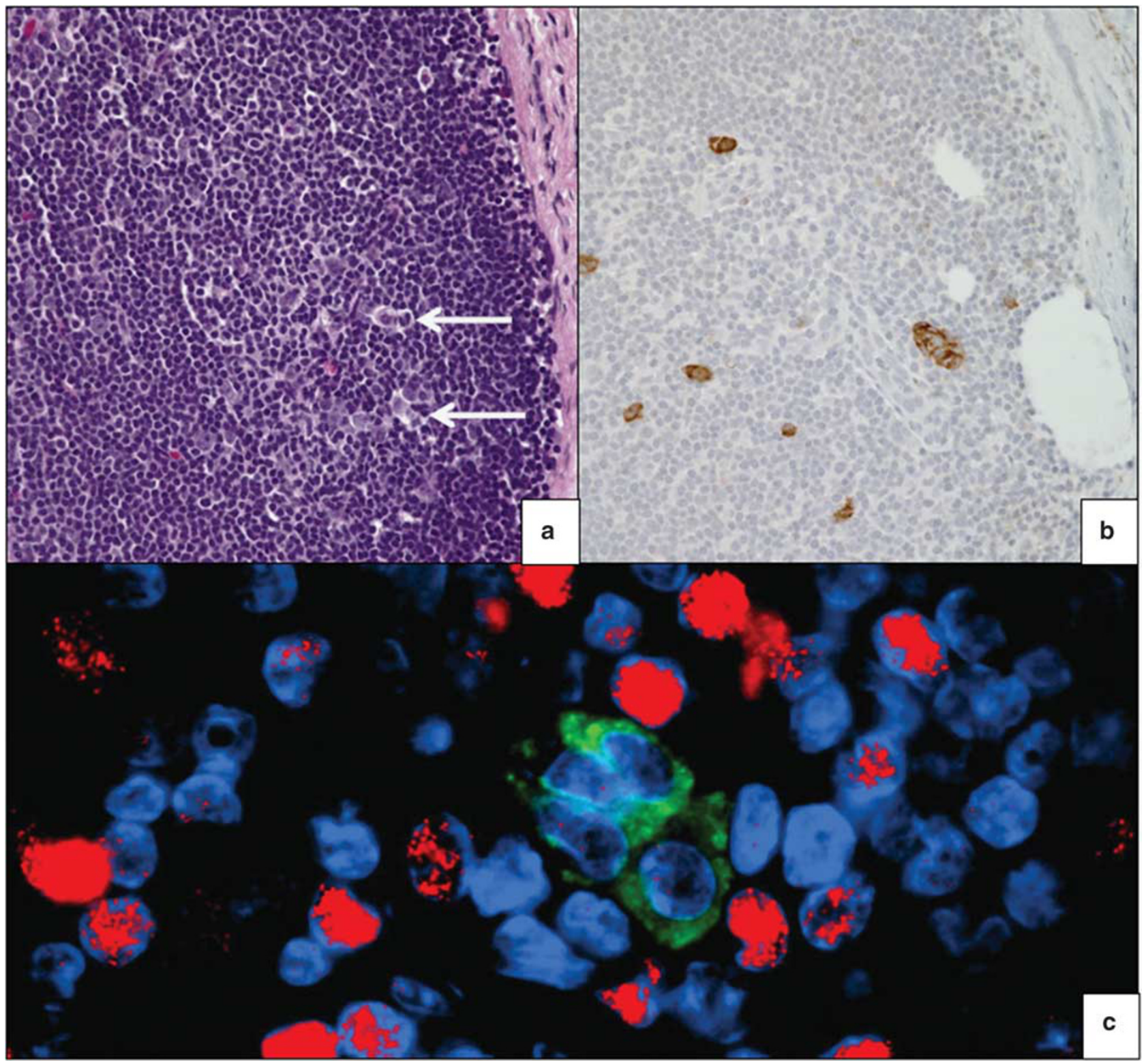

Figure 3 Case 1: suspicious for intraparenchymal micrometastasis. (a) Lymph node parenchyma with rare, inconspicuous cells with epithelioid cytomorphology (white arrows; H\&E, $\times 20$ ). (b) Single-label IHC for MART-1 (brown, cytoplasmic) highlights rare, scattered single, and aggregated MART-1-positive cells containing nuclei slightly larger than those of lymphocytes $(\times 20)$. (c) Dual-label DIF for MART-11 (green) and 5-hydroxymethylcytosine (red) demonstrates retention of 5-hydroxymethylcytosine nuclear staining within aggregate of MART-1-positive cells $(\times 100)$.

to have deeper primaries than those with only nodal nevi. This is a finding in keeping with the notion that sentinel lymph node positivity is a barometer that correlates with other prognostic attributes of melanoma virulence such as depth.

Although our compiled histological data indicate that melanomas metastatic to sentinel lymph nodes are characteristically intraparenchymal in location, in contrast to the intracapsular localization of benign nodal nevi, this general rule was far from absolute in our cohort. In fact, one of our cases of nodal nevi demonstrated clear-cut extension into the parenchyma from an intracapsular and intratrabecular location (Figures 5a and b), a histological pattern that is typically worrisome for metastasis. Moreover, the nevus cells within the parenchyma were also larger than those within the capsule and trabeculae. However, 5-hydroxymethylcytosine nuclear expression was retained within these cells (Figure 5c), which is consistent with their cytological benignancy and architecural unity with the more conventional intracapsular/intratrabecular components. This case demonstrates that neither the microanatomic location nor the pattern of spread is 


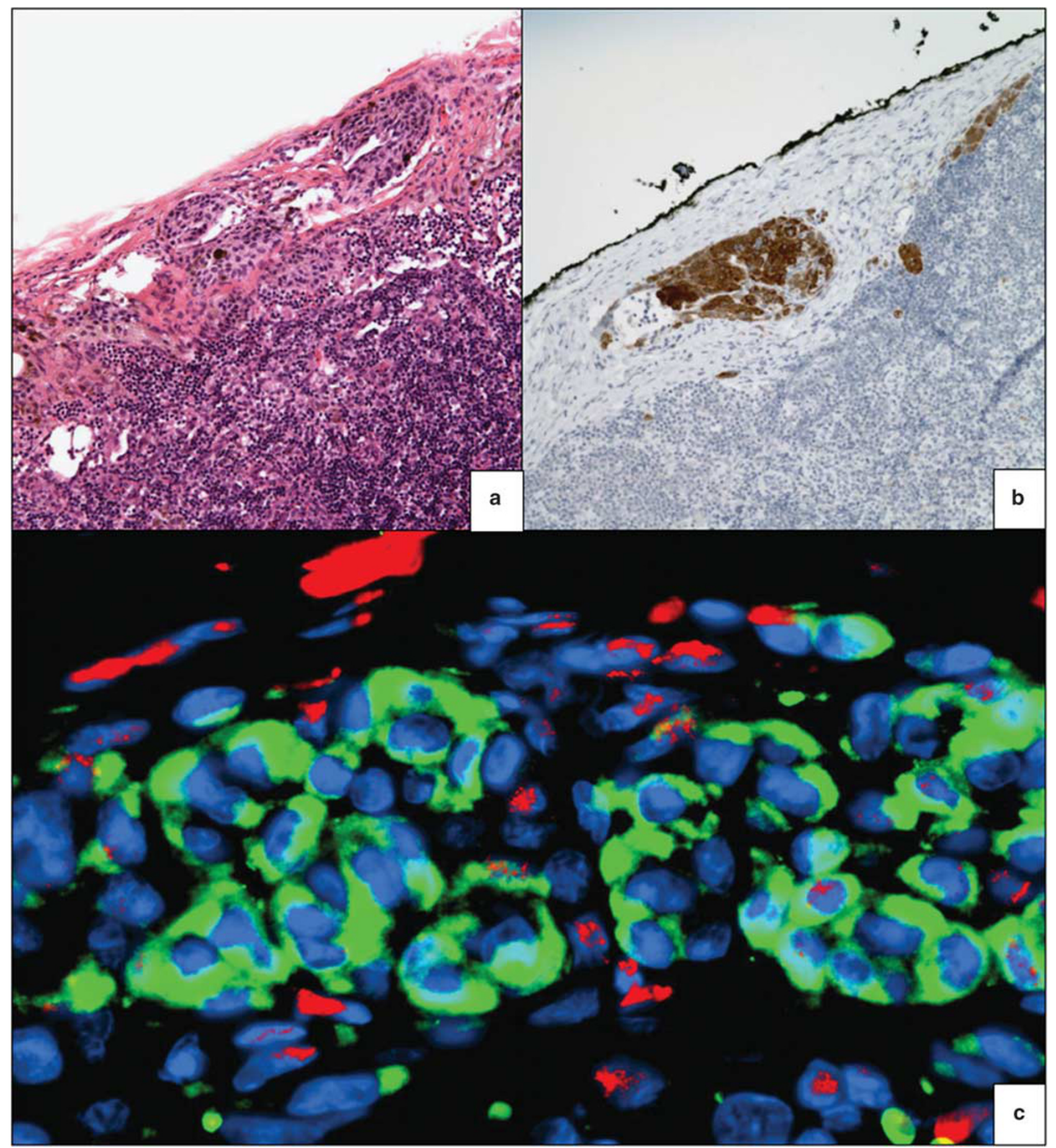

Figure 4 Case 2: suspicious for intracapsular metastatic melanoma. (a) Metastatic melanoma cells present in spatial association with a capsular lymphovascular space $(H \& E, \times 20)$. (b) Single-label IHC for MART-1 (brown) highlights MART-1-positive cells within the capsule and scattered subcapsular cells $(\times 20)$. (c) Dual-label DIF for MART-1 (green) and 5-hydroxymethylcytosine (red) demonstrates loss of 5-hydroxymethylcytosine nuclear expression within MART-1-positive cells, consistent with metastatic melanoma ( $\times 100)$.

sufficient, in itself, to distinguish true metastatic disease from nodal nevi. Our data also indicate that the size of the focus and the overall cytomorphology may, in some cases, also be inadequate for making a definitive distinction. The new AJCC guidelines additionally classifies patients having microscopic nodal metastases, even when only a single cell is identified, as stage III, based on data that associated such findings with reduced overall survival. ${ }^{1}$ Moreover, recent clinical data further support that 


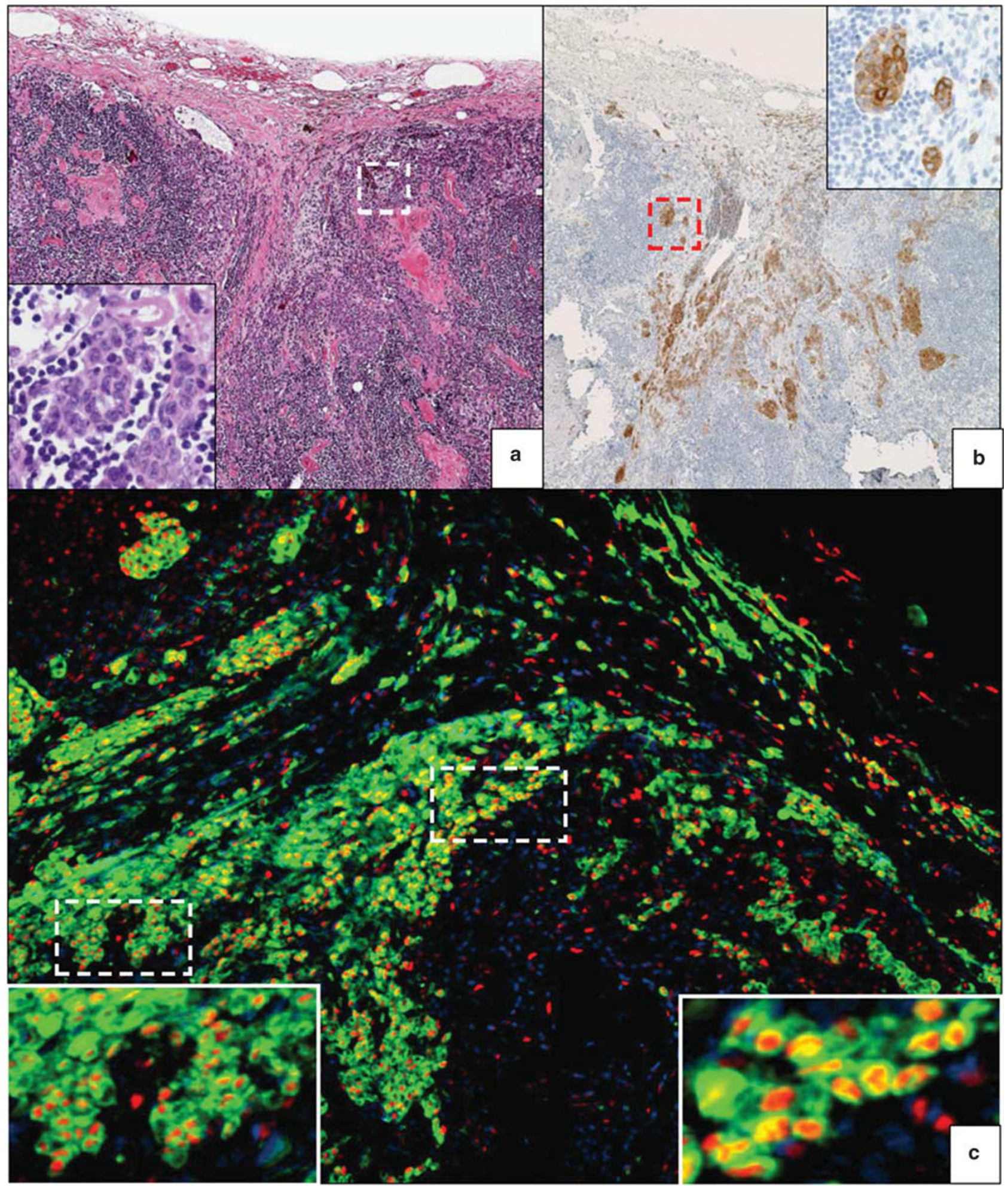

Figure 5 Intracapsular nodal nevus with extension into the parenchyma. (a) H\&E $(\times 10)$. Inset: high-power view of enclosed region of bland, nevic cytomorphology. (b) Single-label IHC for MART-11 (brown) highlights intraparenchymal collections of MART-1-positive cells ( $\times 10$ ). Inset: high-power view of nests of MART-1-positive cells also within lymph node parenchyma. (c) Dual-label DIF for MART11 (green) and 5-hydroxymethylcytosine (red) highlights retention of nuclear 5-hydroxymethylcytosine staining within MART-1-positive cells within the capsule and within the lymph node parenchyma, supporting the diagnosis of intracapsular and intraparenchymal nodal nevus $(\times 10)$. Inset (left): high-power view of MART-1- and 5-hydroxymethylcytosine-positive cells extending into lymph node parenchyma. Inset (right): high-power view of intracapsular nevic cells showing high intensity (red-yellow) nuclear 5-hydroxymethylcytosine expression. 


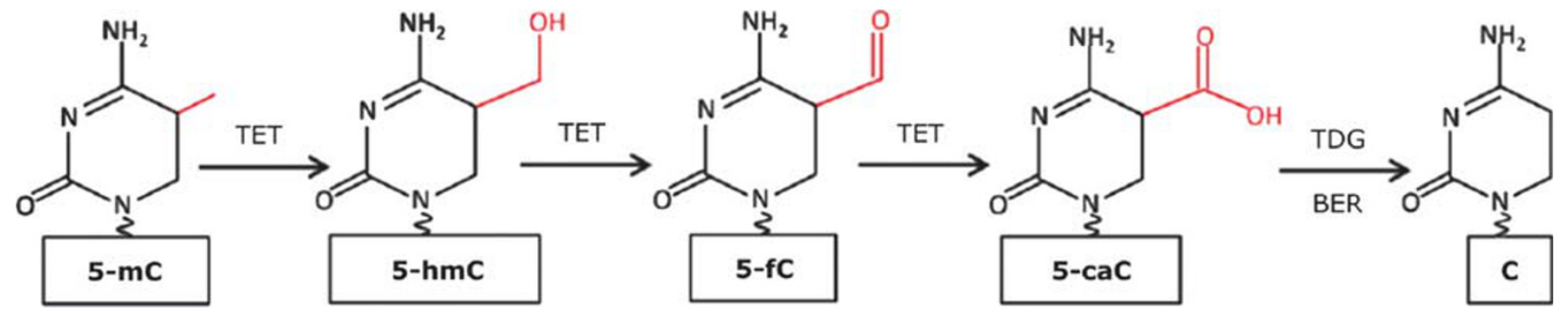

Figure 6 Summary of the active DNA demethylation biochemical pathway. Ten-Eleven Translocase (TET) family dioxygenases catalyze the sequential, iterative oxidation reactions to the methyl group of 5-mC as shown. This critical pathway enables the demethylation of 5 -mC to form the 'unmodified' DNA pyrimidine base, cytosine, providing a mechanism for maintaining the fidelity of DNA methylation patterns. 5-mC, 5-methylcytosine; 5-hmC,5-hydroxymethylcytosine; 5-fC, 5-formylcytosine; 5-caC, 5-carboxylcytosine; BER, base excision repair; C, cytosine; TDG, thymine DNA glycosylase.

even very small numbers of melanoma cells in sentinel lymph nodes may impact a patient's prognosis. ${ }^{17}$ In light of these recent findings and recommendations, adjunctive strategies to assist in the accurate detection of sentinel lymph node metastasis and their distinction from benign nodal nevi or isolated nevic cells are critical.

In the present study, we have demonstrated through immunohistochemistry and direct immunofluorescence that the epigenetic mark 5-hydroxymethylcytosine distinguishes benign nodal nevi from metastatic melanoma in sentinel lymph node biopsies. Our findings are in keeping with prior and more recent corroborative studies of 5-hydroxymethylcytosine expression in primary skin sections containing either benign nevi or malignant melanoma. ${ }^{12,13,18}$ The loss of 5-hydroxymethylcytosine is now a well-documented 'epigenetic hallmark' of human malignancy, and has been shown to be an independent predictor of worse prognosis in melanoma as well as a number of other cancers. ${ }^{13,19-22}$ The absence of 5-hydroxymethylcytosine is thought to reflect dysfunction of the tumor-suppressive TET family of enzymes and the active DNA demethylation pathway (Figure 6). Perturbation or dysfunction to this critical pathway is thought to facilitate global epigenomic instability and may be a critical feature of malignancy. We have previously reported a histopathological corollary to this basic pathobiology, namely that increasing loss of nuclear 5-hydroxymethylcytosine expression parallels increasing degrees of melanocytic dysplasia and that this staining pattern also tightly correlates with increasing nuclear diameter. ${ }^{12}$

From a strictly biological standpoint, we took interest in the modestly higher sensitivity and specificity of 5-hydroxymethylcytosine in distinguishing metastatic melanoma from nodal nevi in sentinel lymph node biopsies compared with previously reported markers. ${ }^{5}$ Immunohistochemical investigation of 5-hydroxymethylcytosine expression in normal human tissues has demonstrated that regenerative, stem-like basal epithelia consistently shows 'loss of 5-hydroxymethylcytosine' similar to their malignant counterparts. ${ }^{19}$ Thus, the corresponding absence of TET enzyme and active DNA demethylation function may be a reflection of a more undifferentiated, 'stem-like' epigenomic cellular state. We have previously reported that nestin and SOX2, markers of neural crest progenitor stem cells, are co-expressed in metastatic melanomas but much less commonly so in benign nevi. ${ }^{10}$ The recently reported expression of nestin and SOX2 in metastatic melanomas within sentinel lymph node specimens may, similar to the 'loss of 5-hydroxymethylcytosine', also reflect a 'less'-differentiated state, allowing for its distinction from nodal nevi. However, we hypothesize that the loss of 5-hydroxymethylcytosine and corresponding TET enzyme dysfunction may more directly reflect the 'upstream' epigenomic milieu of the undifferentiated, versatile cellular biology associated with a malignant, stemlike state that enables the re-expression of previously silenced markers, such as nestin and SOX2. In addition, our previous studies have demonstrated that 'loss of 5-hydroxymethylcytosine' appears to be more prominent in primary cutaneous melanomas that exhibit more aggressive behavior. ${ }^{13}$ Therefore, 5-hydroxymethylcytosine may be even more informative when used to evaluate for metastatic disease. For these reasons, we speculate that 5-hydroxymethylcytosine may be more sensitive and specific for differentiating between benign and malignant melanocytic cells, particularly subsequent to metastatic spread of the latter to sentinel lymph nodes. Certainly, additional studies and further exploration of these proposed mechanisms and pathways are necessary before definitive conclusions can be drawn.

Interestingly, our results indicate a high concordance between the initial histopathological diagnosis and the corresponding 5-hydroxymethylcytosine staining profiles. Our investigation of two 'equivocal' biopsies where a melanoma diagnosis was favored, but could not be definitively rendered, illustrates the potential utility of immunohistochemical staining with 5-hydroxymethylcytosine as a confirmatory test in diagnostically challenging scenarios. However, further immunohistochemical investigation of larger numbers of truly 'equivocal' 
sentinel lymph node biopsies, in concert with supporting clinical and histological annotation, is warranted in order to further test the routine clinical utility of distinguishing markers such as 5-hydroxymethylcytosine. It is noteworthy that in case 1, rare, scattered MART-1-positive cells were present within the lymph node parenchyma (Figures 3a and b). Such a collection could potentially represent either MART-1-positive cells of 'indeterminate lineage $^{\prime 23}$ or true, 'single cell' micrometastasis. The 'scattered' pattern of spread may favor metastatic disease, but such a feature is not definitive or pathognomonic. The loss of 5-hydroxymethylcytosine staining in this case supports the suspicion that this focus represented micrometastatic disease (Figure 3c). In addition, case 2 illustrated a scenario wherein the MART-1-positive cells were predominantly intracapsular in location, a feature that is typically ascribed to nodal nevi (Figures $4 \mathrm{a}$ and b). The suspicion for metastasis in this case was raised based on the overall cytology and possible presence of some of these cells around and within an intracapsular lymphovascular space. Indeed, 5-hydroxymethylcytosine loss within these cells provides further confirmation (Figure 4c).

Given the sentinel lymph node recommendations recently put forth for the evaluation of metastatic melanoma by the AJCC, the volume of sentinel lymph node biopsies encountered in practice will likely increase. This is further compounded by the increasing incidence of malignant melanoma, and the growing hope that precision in staging as well as prophylactic removal of draining nodal basins upon detection of melanoma in sentinel lymph nodes will translate into improved patient care, quality of life, and overall outcome. The data reported herein raises the possibility that diagnostic refinements using functionally relevant and novel epigenetic biomarkers such as 5-hydroxymethylcytosine may assist in obtaining a more precise and reproducible evaluation of sentinel lymph node biopsies in selected melanoma patients.

\section{Acknowledgments}

These results were shared, in part, at the Brigham and Women's Hospital, Massachusetts General Hospital, and Harvard Medical School Department of Pathology Annual Research Celebration (2014) in Boston, MA, USA. This study is supported by NIH grant 5P40CA093683-09 to the SPORE Core at Brigham and Women's Hospital. GFM receives funding from Bristol-Meyers-Squibb for work on melanoma biomarkers (not a funding source for this study).

\section{Disclosure/conflict of interest}

The authors declare no conflict of interest.

\section{References}

1 Piris A, Mihm MC Jr., Duncan LM. AJCC melanoma staging update: impact on dermatopathology practice and patient management. J Cutan Pathol 2011;38: 394-400.

2 Carson KF, Wen DR, Li PX, et al. Nodal nevi and cutaneous melanomas. Am J Surg Pathol 1996;20: 834-840.

3 Fontaine D, Parkhill W, Greer W, et al. Nevus cells in lymph nodes: an association with congenital cutaneous nevi. Am J Dermatopathol 2002;24:1-5.

4 Gambichler T, Scholl L, Stucker M, et al. Clinical characteristics and survival data of melanoma patients with nevus cell aggregates within sentinel lymph nodes. Am J Clin Pathol 2013;139:566-573.

5 Chen PL, Chen WS, Li J, et al. Diagnostic utility of neural stem and progenitor cell markers nestin and SOX2 in distinguishing nodal melanocytic nevi from metastatic melanomas. Mod Pathol 2013;26:44-53.

6 Holt JB, Sangueza OP, Levine EA, et al. Nodal melanocytic nevi in sentinel lymph nodes. Correlation with melanoma-associated cutaneous nevi. Am J Clin Pathol 2004;121:58-63.

7 Mihic-Probst D, Saremaslani P, Komminoth P, et al. Immunostaining for the tumour suppressor gene p16 product is a useful marker to differentiate melanoma metastasis from lymph-node nevus. Virchows Arch 2003;443:745-751.

8 Blokhin E, Pulitzer M, Busam KJ. Immunohistochemical expression of p16 in desmoplastic melanoma. J Cutan Pathol 2013;40:796-800.

9 Dalton SR, Gerami P, Kolaitis NA, et al. Use of fluorescence in situ hybridization (FISH) to distinguish intranodal nevus from metastatic melanoma. Am J Surg Pathol 2010;34:231-237.

10 Laga AC, Zhan Q, Weishaupt C, et al. SOX2 and nestin expression in human melanoma: an immunohistochemical and experimental study. Exp Dermatol 2011;20:339-345.

11 Girouard SD, Laga AC, Mihm MC, et al. SOX2 contributes to melanoma cell invasion. Lab Invest 2012;92: 362-370.

12 Larson AR, Dresser KA, Zhan Q, et al. Loss of 5-hydroxymethylcytosine correlates with increasing morphologic dysplasia in melanocytic tumors. Mod Pathol 2014;27:936-944.

13 Lian CG, Xu Y, Ceol C, et al. Loss of 5-hydroxymethylcytosine is an epigenetic hallmark of melanoma. Cell 2012;150:1135-1146.

$14 \mathrm{Wu} \mathrm{H}$, Zhang Y. Reversing DNA methylation: mechanisms, genomics, and biological functions. Cell 2014;156:45-68.

15 Williams K, Christensen J, Helin K. DNA methylation: TET proteins-guardians of CpG islands? EMBO Rep 2012;13:28-35.

16 Coit DG, Andtbacka R, Anker CJ, et al. Melanoma, version 2.2013: featured updates to the NCCN guidelines. J Natl Compr Canc Netw 2013;11: 395-407.

17 Ulmer A, Dietz K, Hodak I, et al. Quantitative measurement of melanoma spread in sentinel lymph nodes and survival. PLoS Med 2014;11:e1001604.

18 Uchiyama R, Uhara H, Uchiyama A, et al. 5-Hydroxymethylcytosine as a useful marker to differentiate between malignant melanomas and benign melanocytic nevi. J Dermatol Sci 2014;73:161-163. 
19 Haffner MC, Chaux A, Meeker AK, et al. Global 5-hydroxymethylcytosine content is significantly reduced in tissue stem/progenitor cell compartments and in human cancers. Oncotarget 2011;2:627-637.

20 Yang Q, Wu K, Ji M, et al. Decreased 5-hydroxymethylcytosine $(5-\mathrm{hmC})$ is an independent poor prognostic factor in gastric cancer patients. J Biomed Nanotechnol 2013;9:1607-1616.

21 Liu C, Liu L, Chen X, et al. Decrease of 5-hydroxymethylcytosine is associated with progression of hepatocellular carcinoma through downregulation of TET1. PloS ONE 2013;8:e62828.

22 Orr BA, Haffner MC, Nelson WG, et al. Decreased 5-hydroxymethylcytosine is associated with neural progenitor phenotype in normal brain and shorter survival in malignant glioma. PloS ONE 2012;7:e41036.

23 Satzger I, Volker B, Meier A, et al. Prognostic significance of isolated HMB45 or Melan A positive cells in Melanoma sentinel lymph nodes. Am J Surg Pathol 2007;31:1175-1180. 